\title{
Due di noi
}

\section{Two of us}

Giovanni Lodi

Dipartimento di Scienze Biomediche, Chirurgiche e Odontoiatriche

Università degli Studi di Milano

via Beldiletto 1/3 Milano 20142

Italia

giovanni.lodi@unimi.it

tel. +390250319021

fax. +390250319041

Maria è laureata da un paio d'anni. II centro low-cost in cui lavora il mercoledì e il venerdì non è esattamente quello che aveva in mente la mattina del test di amissione. Ma è meglio del service dove fa conservativa il martedì, e comunque la pagano puntualmente, non come il titolare dello studio con cui collabora il lunedì. E poi di questi tempi guai ad essere choosy.

Maria viene pagata a percentuale, ma i prezzi del centro sono molto low, così la fattura che stacca a fine mese non è sufficiente a lasciare la casa dei suoi. Ma se in autunno dovesse entrare a ortodonzia, tutto cambierà in meglio. $\mathrm{O}$ almeno spera.

Maria oggi deve preparare un moncone, è una delle prime volte, e dopo un quarto d'ora di lavoro il risultato è inadeguato, come il tempo e le frese che ha a disposizione. Meglio andare a chiedere aiuto a Mario, che lavora nel riunito a fianco.

Mario di monconi ne ha preparati a centinaia dal giorno in cui si è specializzato in odontostomatologia. Un mondo fa, e sono passati meno di trent'anni.

Mario viene pagato all'ora. Forse un compenso a percentuale gli permetterebbe di guadagnare di più, perché è un bravo protesista, ma a lui oggi serve sicurezza. Quella che ha perso quando la crisi e un centro simile a quello in cui lavora adesso lo hanno costretto a chiudere lo studio. Fortunatamente ha saldato tutti i debiti, tranne qualche pendenza con l'agenzia delle entrate.

Mario spera che i finanziatori del centro low-cost continuino a fare buoni affari. E intanto conta gli anni che lo separano dalla pensione. Però fare il dentista gli piace ancora molto e insegnare il mestiere alla giovane Maria lo fa stare bene.

Buona lettura. 